\title{
A Transurethral Prostate Resection Manipulator for Minimal Damage to Mucous Membrane
}

\author{
Ryuji Hashimoto, Daeyoung Kim, Nobuhiko Hata, and Takeyoshi Dohi \\ Department of Mechano-Informatics, Graduate School of Information Science \\ The University of Tokyo, 7-3-1 Hongo Bunkyo-Ku, Tokyo, 133-8656, Japan \\ \{hashimoto, young, noby, dohi\}@atre.t.u-tokyo.ac.jp \\ http://www.atre.t.u-tokyo.ac.jp/index.html
}

\begin{abstract}
Transurethral Resection of the Prostate (TUR-P) is the most common surgical procedure for Benign Prostatic Hyperplasia (BPH). However, damage to the mucous membrane of the urethra and extended surgery lead to complications. In order to resolve these problems, we propose a new prostatectomy, and develop a TUR-P manipulator, which has a prostate displacement mechanism and a continuous perfusion-resection mechanism. Using these mechanisms, our manipulator can remove enlarged tissue rapidly through small incision on urethra, thus minimizing damage to the mucous membrane. In the experiments, $640 \mathrm{gf}$ of power was delivered to the bending mechanism. The bending motion was highly repeatable, to within \pm 0.4 degrees. The device removed material from a swine-tissue test sample at $0.7 \mathrm{~g} / \mathrm{min}$. These results showed the machine's groundbreaking capability to shorten surgery times by one hour, from 90 to 30 minutes.
\end{abstract}

\section{Introduction}

Benign prostatic hyperplasia (BHP) is a common disease among older men. As a man ages, the prostate becomes enlarged. Since the prostate surrounds the urethra, it places pressure on the urethra. This makes urination difficult and leads to dysfunctions of the bladder and kidney. More than half of all men in their 60s, and as many as 80 percent of men in their 70s and 80s, have some symptoms of $\mathrm{BPH}[1,2$.

Transurethral resection of the prostate (TUR-P) is a common cure for BPH, and provides a less invasive alternative to open prostatectomy. In the TUR-P procedure, the surgeon inserts a resectoscope through the penis, and cuts the prostate tissue into pieces with an electrical loop during the 90 -minute operation. The pieces of tissue are carried by the perfusate into the bladder and then flushed out at the end of the operation. After surgery, the removed tissue is routinely checked for hidden cancer cells [3]4].

However, complications are possible with TUR-P. TUR syndrome, which leads to dizziness and nausea, occurs with absorption of perfusate in extended surgery. Urinary tract infection (UTI) occurs with damage to the mucous membrane of the urethra during cutting of an enlarged prostate [56]. 
To avoid the TUR-P complications, some devices have been proposed by several groups. Davies developed a manipulator for TUR-P surgery under accurate control, with guidance provided by a $3 \mathrm{D}$ model of the prostate generated from the ultrasound images 78 . Accurate and repeatable cutting by the manipulator can prevent bleeding and damage to sphincter muscle and nerves.

Matsumiya has proposed a preliminary design for a prostatectomy cutter which reaches the prostate via a small incision on the urethra, thus minimizing damage to the mucous membrane of the urethra 9 .

Against the above background, we propose a new TUR-P manipulator based on Matsumiya's design theory. Two original approaches are applied to allow the surgeon to remove the prostatic tissue quickly without damaging the mucous membrane of the urethra: a prostate displacement mechanism and a continuous perfusion-resection mechanism. This paper is a reports on 1) the newly developed TUR-P manipulator and 2) evaluation of the bending characteristics and performance in resection.

\section{Methods}

\subsection{System Configuration}

The complete system has three parts: a manipulator, an image-guided system, and a controller (Fig. 1). The manipulator is fixed to a flexible holder (Point Setter, Mitaka USA Inc.). Firstly, the manipulator is inserted into urethra. The flexible holder is then locked, and the surgeon controls the manipulator with guidance in the form of an ultrasound image. All apparatus of the TUR-P manipulator is surrounded by the dashed line in Fig. [1]

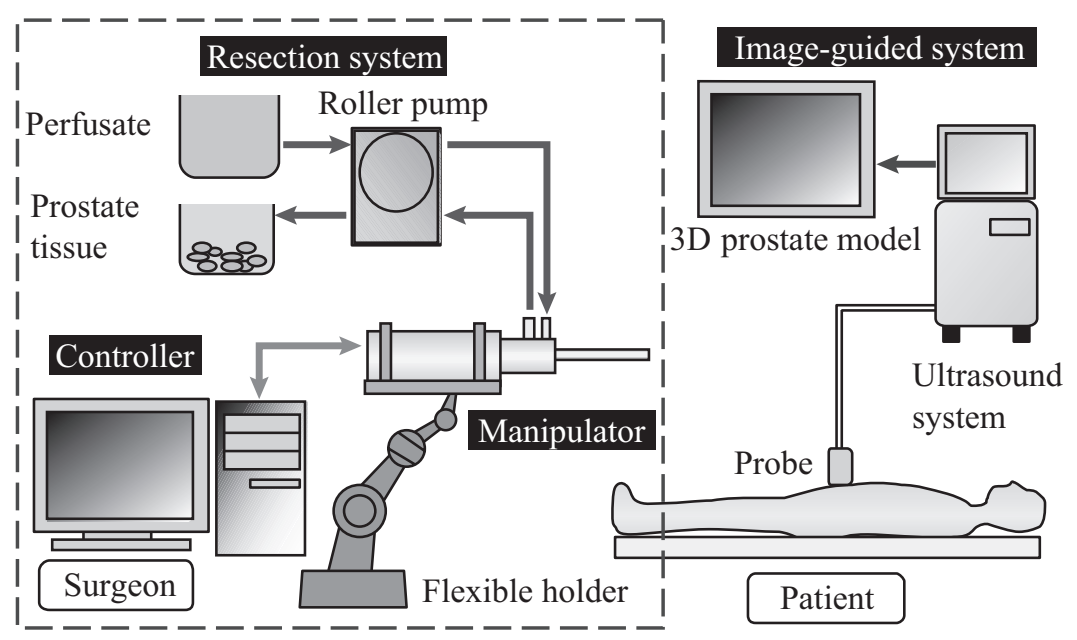

Fig. 1. Conceptual model of the complete system 


\subsection{Bending and Resection Mechanism}

Figure 2(a) shows the bending mechanism that displaces the prostate to prevent damage to the mucous membrane of the urethra. This mechanism is based on Matumiya's manipulator [9. We equipped the manipulator's end effector with an arm and a cutter. Firstly, the manipulator is inserted through the urethra. Then the arm bends to move the enlarged prostate, and the cutter is inserted into the prostate to remove prostate tissue by cutting. While the cutter cuts the enlarged tissue through linear motion, the arm can move to any angle. The possibility of incision in the mucous membrane can thus be restricted to a single point.

Organs such as the bladder and sphincter muscle are situated around the prostate, and should not be damaged. We thus attach a balloon catheter to the end of the arm. When the balloon is inflated, it holds the manipulator steady against the urethra, preventing damage to these organs.

Figure 2(b) shows the continuous perfusion-resection mechanism for rapid resection. The cutter is equipped with a drill and a perfusion pipe. While the cutter is inserted into the prostate, the drill continues to cut the enlarged tissue into small piece. A roller pump drives perfusate into the perfusion pipe; the perfusate mixes with the prostate tissue and is aspirated by the pump through the cutter. Through the combination of these mechanisms, the manipulator cuts and removes the enlarged tissue at the same time. This means that less time is required for the surgery.

\subsection{Manipulator Specification}

We developed the manipulator shown in Fig. [3. The manipulator has three parts: an end effector, a driving unit and a connection mechanism.

The end effector has an arm, a cutter and a resection system that includes a drill and a perfusion pipe (Fig. 4 left panel). The arm is $6 \mathrm{~mm}$ in diameter and

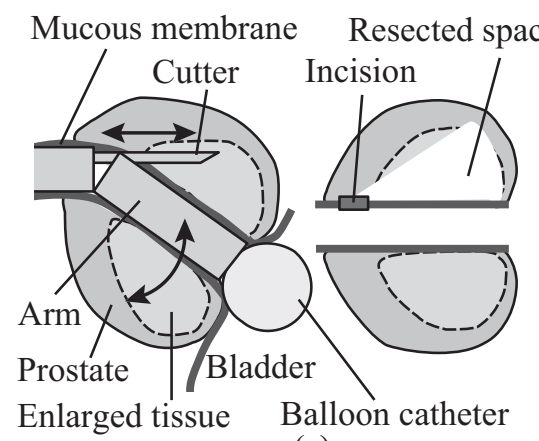

(a)

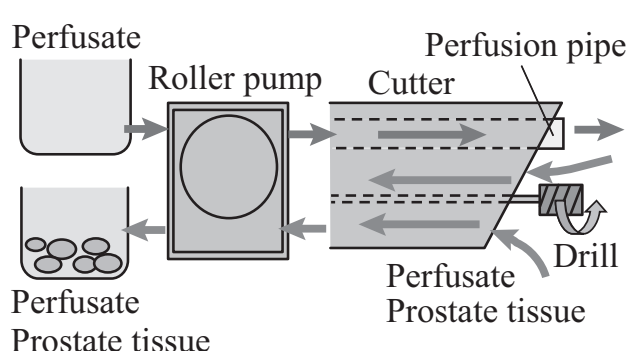

(b)

Fig. 2. (a) Prostate displacement mechanism for minimizing damage to the mucous membrane (b) Continuous perfusion-resection mechanism for rapid prostatectomy 


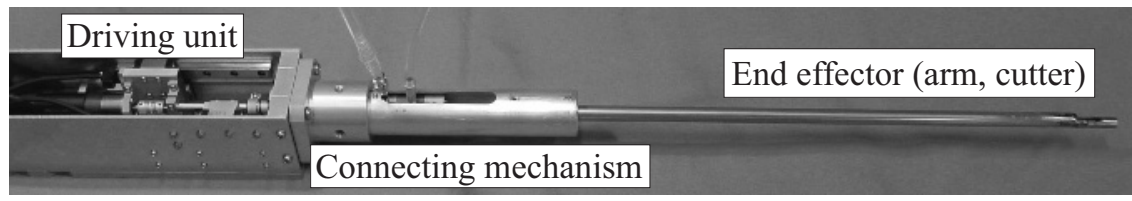

Fig. 3. Whole body of the manipulator, including end effector, driving unit, and connection mechanism

20-mm long. The arm can be bent through a range of 0 to 45 degrees, with a resolution of 0.1 degrees. The diameter of the cutter is $3.5 \mathrm{~mm}$. Its stroke length is $35 \mathrm{~mm}$, with a resolution of $0.1 \mathrm{~mm}$. The diameter of the cover sheath, which is inserted in the urethra, is $8 \mathrm{~mm}$. This is the same size as the resectoscope. These elements are demountable and sterilizable. In order to accurately bend the enlarged prostate, we adopted a slider linkage mechanism for bending (Fig. \$ right panel), because it realizes greater stiffness and less backlash than a wiredriven system. When the actuator pushes or pulls link1, link2 bends or extends the arm.
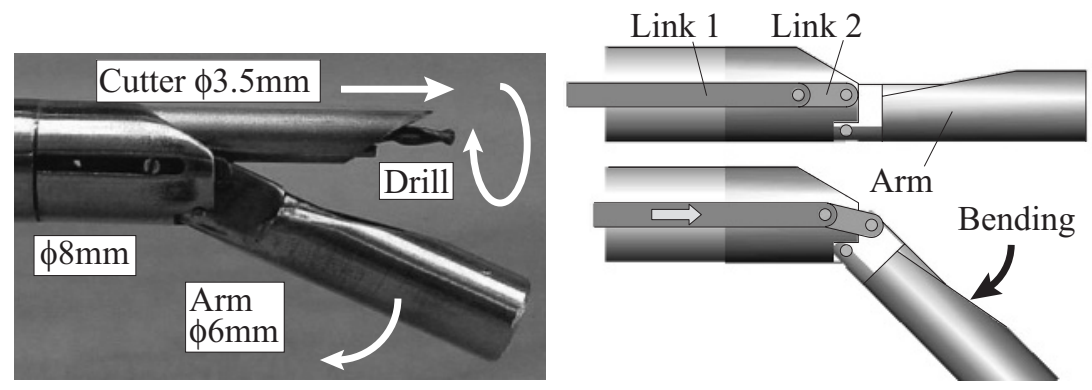

Fig. 4. End effector (left) and slider linkage mechanism (right)

The driving unit consists of motors, photo-sensors and ball screws. The rotational velocity and direction of the motor is controlled from a console. The ball screws transform rotation of the motors into translation. The photo-sensors are used to tune the zero-point of the arm and cutter. These elements are unsterilizable, so the driving unit has to be covered by a drape during the operation. The size of the driving unit is $44 \times 44 \times 180 \mathrm{~mm}$.

The connection mechanism shown in Fig. 5 provides fixture-free connection of the sterilizable end effector and the unsterilizable driving unit. We equipped the connector with a coupling for drill rotation, and springs for translation of the arm and cutter translation. The arm bends in response to the driving unit pushing the arm link. The arm extends when the spring pulls the arm link. The cutter is moved by the same mechanism as the arm. 

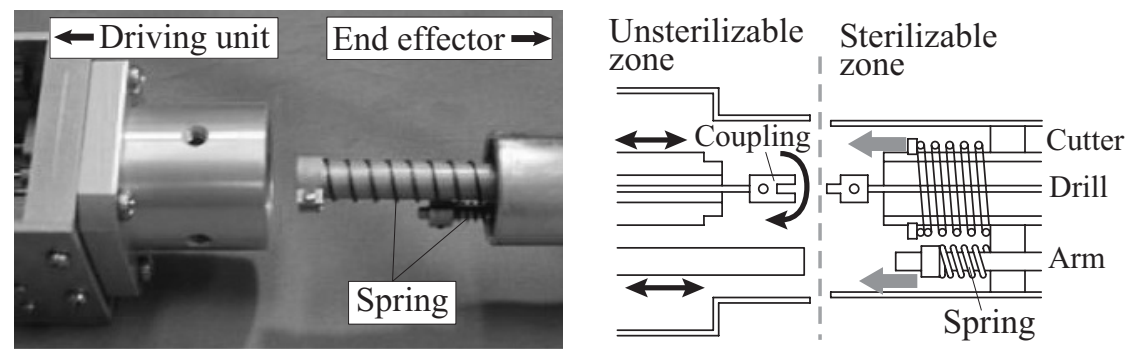

Fig. 5. Connection mechanism for sterilization and quick attachment

\section{Evaluation Study and Results}

\subsection{Bending Mechanism}

We measured the force generated by the arm's bending motion. The result was $640 \mathrm{gf}$. It is sufficient to displace the enlarged prostate which weighs $40 \mathrm{~g}$ on average. We then evaluated the characteristics of the bending movement of the arm in the unloaded state and carrying a weight of $500 \mathrm{gf}$. The manipulator arm is bent through the range from 0 to 45 degrees and then extended 45 to 0 degrees. The results are shown in Fig. [6.

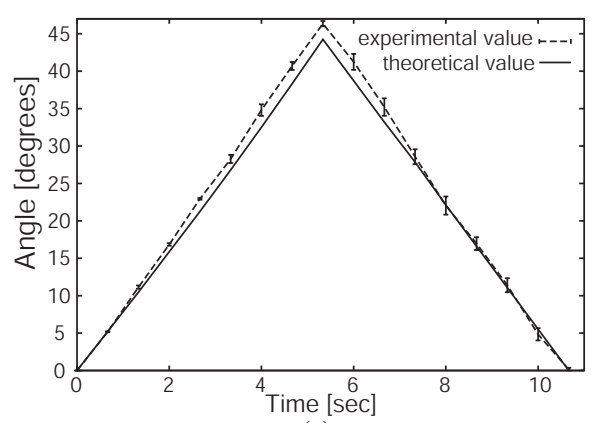

(a)

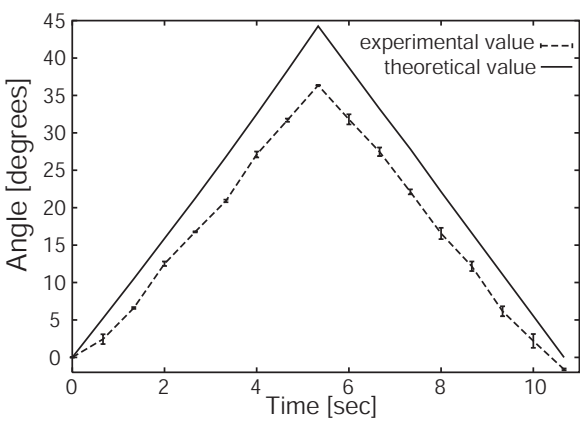

(b)

Fig. 6. Bending characteristics: (a) nonloaded and (b) loaded with a 500 gf weight

In the unloaded state, the experimental values were always greater than the theoretical values. When the theoretical value was 45 degrees, the experimental value was $46.5 \pm 0.3$ degrees. The average repeatable accuracy was \pm 0.8 degrees.

With the 500 gf weight, the experimental values were always lower than the theoretical values. When the theoretical value was 45 degrees, the experimental value was $36.3 \pm 0.2$ degrees. The repeatable accuracy of bending motion was \pm 0.4 degrees. 


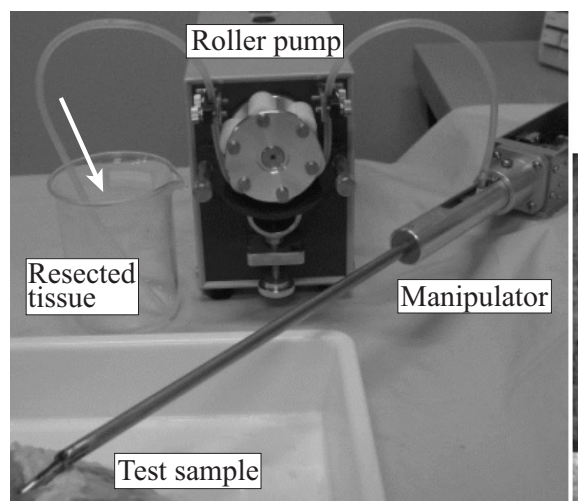

(a)

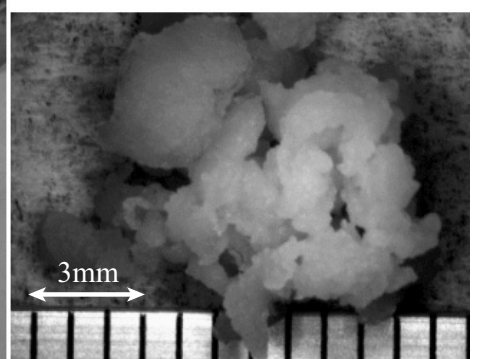

(b)

Fig. 7. (a) Equipment for the resection experiment. (b) Resected tissue of the test sample

\subsection{Performance in Resection}

We evaluated the performance of the manipulator in resection. In this example, we used swine thigh muscle as a test sample. We measured the resected weight of the test sample while the manipulator continued to insert the cutter, drill the tissue and remove it by pumping for one minute (Fig. 7.a). The inserted cutter's stroke was $25 \mathrm{~mm}$. Parameters were the drill's rotational velocity $\omega$ and the velocity of cutter insertion $v$. Fig. $7 \mathrm{~b}$ shows the resected tissue of the sample. The sample tissue was mixed with perfusate, and removed by the pump as material suspended in fluid.

In conventional TUR-P surgery, surgery takes 90 minutes and the resected prostates weigh $20 \mathrm{~g}$ on average, so the efficiency of resection is $0.2 \mathrm{~g} / \mathrm{min}$. Firstly, we set $v$ to a constant $15 \mathrm{~mm} / \mathrm{sec}$ and changed $\omega$ from 250 to $1250 \mathrm{rpm}$ (Fig. 8]a). Although the resected weight increased with $\omega$ increased, there was little difference between the effect at $610 \mathrm{rpm}$ and at $1250 \mathrm{rpm}$. We thus set $\omega$ to a constant $610 \mathrm{rpm}$ and changed $v$ from 10 to $20 \mathrm{~mm} / \mathrm{sec}$ (Fig. 8. b). The resected weight reached its peak of $0.7 \mathrm{~g} / \mathrm{min}$ at $15 \mathrm{~mm} / \mathrm{sec}$. Little tissue was resected with the machine operating at less than $10 \mathrm{~mm} / \mathrm{sec}$ or more than $20 \mathrm{~mm} / \mathrm{sec}$.

\section{Discussion}

We have developed a TUR-P manipulator that minimizes damage to the mucous membrane of the urethra and shortens the time taken by surgery.

We confirmed that the bending motion of our manipulator was highly repeatable and delivered sufficient power to the arm. The error in the bending angle with the load of $500 \mathrm{gf}$ may be caused by straining of the arm. The 500-gf load is transmitted into a 3300-gf thrust against a link of the arm. In any case, we can cancel out this error by calibration in which we use experimental values. 


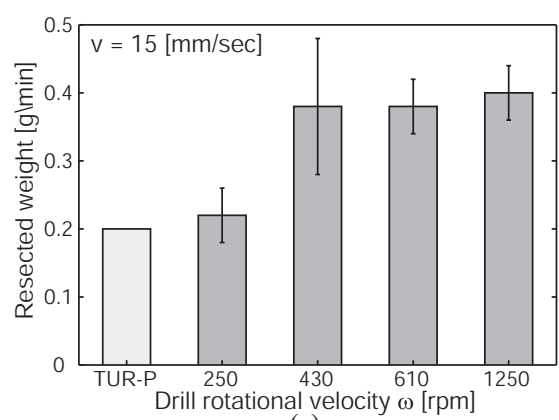

(a)

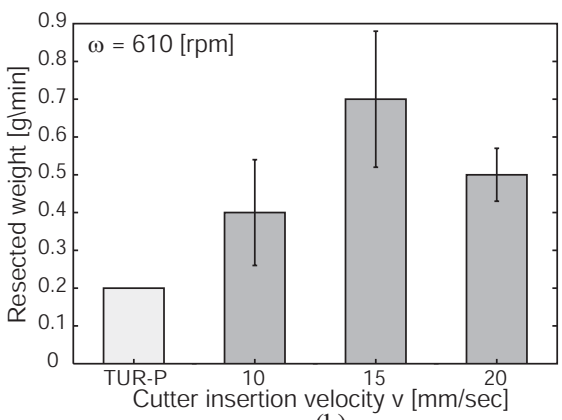

(b)

Fig. 8. Resected weight with changing (a) the drill rotational velocity $\omega$, and (b) the cutter insertion velocity $v$

These bending characteristics are such that our manipulator is capable of precisely displacing an enlarged prostate, so we can expect the device to prevent damage to the mucous membrane of the urethra

The resection experiments confirmed that our manipulator is capable of removing sufficient volume from a test sample in a short enough time. In these conditions, our manipulator can finish removing the target weight (an average of $20 \mathrm{~g}$ ) from an enlarged prostate within 30 minutes. Conventional TUR-P surgery takes 90 minutes; our manipulator thus cuts one hour from the time taken by surgery. We can expect this to reduce the possibility of TUR syndrome. After the resection experiment, we were able to confirm the usefulness of the connection mechanism for sterilization and cleaning of the end effector. In this experiment, however, we were unable to use a swine prostate, because they are too small for use in evaluation of the resection mechanism's performance. We thus need to remember that there is a difference between the thigh muscle of the test sample and actual prostate tissue, and that the resected weight might vary according to tissue characteristics.

In another respect, the manipulator has a potential as a biopsy tool for prostate cancer; that is, as the tool that removes tissue for examination. Detection of early prostate cancer is an important function because BPH and prostate cancer are strongly related, and cancer cells are found in 10 percent of resected samples after surgery [3].

We are going to add a further degree of freedom so that the whole body of the manipulator becomes rotatable. The manipulator will then be capable of removing from any region of the prostate. Other future work will include the development of an image-guided system in which we use transrectal or transabdominal ultrasonography to identify the locations of the end effector and prostate.

In conclusion, we have developed a TUR-P manipulator that includes a prostate displacement mechanism and continuous perfusion-resection mechanism for TUR-P surgery. We have evaluated the performance of both mechanisms. We 
have confirmed that the bending mechanism had high repeatability and sufficient power was delivered to the arm, and that the resection mechanism was highly efficient. We are sure that our manipulator is capable of accurately displacing an enlarged prostate and removing sufficient prostate tissue in a short time, which leads to a reduced possibility of complications.

\section{References}

1. Berry, S.J., Coffey, D.S., Walsh, P.C., Ewing, L.L.: The Development of Human Benign Prostatic Hyperplasia with Age, Journal of Urology, 132(3) (1984) 474-479

2. Girman, C.J., Jacobsec, S.J., Guess, H.A., Oesterling, J.E., Chute, C.G., Panser, L.A., Lieber, M.M.: Natural History of Prostatism: Relationship among Symptoms, Prostate Volume and Peak Urinary Flow, Journal of Urology, 153(5) (1995) 15101515

3. Koshiba, K., Egawa, S., Ohori, M., Uchida, T., Yokoyama, E., Shoji, K.: Does Transurethral Resection of the Prostate Pose a Risk to Life - 22-Year Outcome, Journal of Urology, 153(5) (1995) 1506-1509

4. Crowley, A.R., Horowitz, M., Chan, E., Macchia, R.J.: Transurethral Resection of the Prostate Versus Open Prostatectomy - Long-term Mortality Comparison, Journal of Urology, 153(3) (1995) 695-697

5. Heide, C., Weninger, E., Ney, L., Sachs, M., Niklas, M., Schmeller, N., Peter, K.: Early Diagnosis of the TUR Syndrome - Ethanol Measurement in Patients with Artificial Respiration, Anasthesiologie Intensivmedizin Notfallmedizin Schmerztherapie, 32(10) (1997) 610-615

6. Vivien, A., Lazard, T., Rauss, A., Laisne, M.J., Bonnet, F.: Infection after Transurethral Resection of the Prostate: Variation among Centers and Correlation with a Long-lasting Surgical Procedure, European Urology, 33(4) (1998) 365-369

7. Mei, Q., Harris, S.J., ArambulaCosio, F., Nathan, M.S., Hibberd, R.D., Wickham, J.E., Davies, B.L.: PROBOT - A Computer Integrated Prostatectomy System, Visualization in Biomedical Computing, 1311 (1996) 581-590

8. Harris, S.J., ArambulaCosio, F., Mei, Q., Hibberd, R.D., Davies, B.L., Wickham, J.E., Nathan, M.S., Kundu, B.: The Probot - an Active Robot for Prostate Resection, Proceedings of the Institution of Mechanical Engineers Part H-Journal of Engineering in Medicine, 211(4) (1997) 317-325

9. Matumiya, K., Masamune, K., Kobayashi, E., Sakuma, I., Dohi, T.: A New Robotic Device for Less Invasive Transurethral Resection of the Prostate, Computer Assisted Radiology and Surgery - CARS2000, (2000) 134-138 